\title{
The metastasis suppressor KISS1 lacks antimetastatic activity in the C8161.9 xenograft model of melanoma.
}

\author{
Jean-Marc Navenot \\ Thomas Jefferson University \\ Barry Evans \\ Thomas Jefferson University \\ Shinya Oishi \\ Kyoto University \\ Shohei Setsuda \\ Korbovluhiseasityadditional works at: https://jdc.jefferson.edu/pacbfp

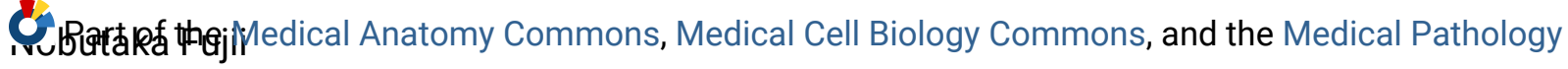 \\ Kuyotonldnisyersity \\ Let us know how access to this document benefits you
}

See next page for additional authors

\section{Recommended Citation}

Navenot, Jean-Marc; Evans, Barry; Oishi, Shinya; Setsuda, Shohei; Fujii, Nobutaka; and Peiper, Stephen C, "The metastasis suppressor KISS1 lacks antimetastatic activity in the C8161.9 xenograft model of melanoma." (2012). Department of Pathology, Anatomy, and Cell Biology Faculty Papers. Paper 89.

https://jdc.jefferson.edu/pacbfp/89

This Article is brought to you for free and open access by the Jefferson Digital Commons. The Jefferson Digital Commons is a service of Thomas Jefferson University's Center for Teaching and Learning (CTL). The Commons is a showcase for Jefferson books and journals, peer-reviewed scholarly publications, unique historical collections from the University archives, and teaching tools. The Jefferson Digital Commons allows researchers and interested readers anywhere in the world to learn about and keep up to date with Jefferson scholarship. This article has been accepted for inclusion in Department of Pathology, Anatomy, and Cell Biology Faculty Papers by an authorized administrator of the Jefferson Digital Commons. For more information, please contact: JeffersonDigitalCommons@jefferson.edu. 


\section{Authors}

Jean-Marc Navenot, Barry Evans, Shinya Oishi, Shohei Setsuda, Nobutaka Fujii, and Stephen C Peiper 


\section{As submitted to:}

\section{Melanoma research}

\section{And later published as:}

The Metastasis Suppressor KISS1 Lacks Anti-Metastatic Activity in the

\section{C8161.9 Xenograft Model of Melanoma}

\section{Volume 22, Issue 2, April 2012, pp.140-150. \\ DOI: 10.1097/CMR.0b013e328350fa07}

Running head: KISS1 Lacks Anti-Metastatic Activity in the C8161.9 Model

Jean-Marc Navenot ${ }^{1}$, Barry Evans ${ }^{1}$, Shinya Oishi ${ }^{2}$, Shohei Setsuda ${ }^{2}$, Nobutaka Fujii ${ }^{2}$ and Stephen C. Peiper ${ }^{1}$

${ }^{1}$ Department of Pathology, Anatomy and Cell Biology, Thomas Jefferson University, Philadelphia, PA 19107

${ }^{2}$ Graduate School of Pharmaceutical Sciences, Kyoto University, Sakyo-ku, Kyoto 6068501, Japan

Corresponding author: Jean-Marc Navenot, Department of Pathology, Anatomy and Cell Biology, Thomas Jefferson University, 1020 Locust Street, Room JAH 336C, Philadelphia, PA 19107. Tel. 215503 6150, Fax 2155035929. 
Request for reprints: Jean-Marc Navenot

Financial support: This publication was made possible by Grant Number SAP\#4100047652 from the Commonwealth of Pennsylvania - Department of Health.

Conflict of interest: none declared 


\begin{abstract}
Ojectives: Use the established xenograft model of human melanoma C8161.9 to test a pharmacological approach to effect of the metastasis suppressor KISS1.

Methods: A KISS1 analog was used to inhibit the metastatic development of C8161.9 cells in nude mice. Further experiments were performed to test the validity of the C8161.9 model and test the connection between KISS1 expression and loss of metastatic potential. New clones of C8161.9 cells were obtained, with or without KISS1 expression, and were tested for metastasis formation.
\end{abstract}

Results: The absence of benefit in survival with the KISS1 analog compared to PBS prompted us to revisit the C8161.9 model. We found that the cells expressing KISS1 used in the previous study and obtained by transfection and single cell cloning were defective for formation of both orthotopic tumor and metastases. In mixing experiments, these cells could not suppress orthotopic tumor growth of KISS1-negative C8161.9 cells, suggesting that the suppression of metastasis by C8161.9-KISS1 cells may be intrinsic to the selected clone rather than related to KISS1 expression. Isolation in soft agar of clones from parental C8161.9 cells yielded cell populations that phenotypically and genotypically mimicked the KISS1-positive clone. Also, new clones expressing KISS1 did not show any decrease in metastatic growth.

Conclusions: These data demonstrate the heterogeneity of cell types in the C8161.9 cell line and the high risk of artifact linked to single cell selection. A different xenograft model will be necessary to evaluate the use of KISS1 analogs as anti-metastatic therapy.

Keywords: metastasis; metastasis suppressor; KISS1; GPR54; cell line 


\section{Introduction}

KISS1 was identified as a metastasis suppressor gene in human malignant melanoma cells $[1,2]$. As opposed to tumor suppressors, metastasis suppressors do not inhibit the growth of primary tumors, but specifically target the spread of tumor cells to distant organs and the establishment of secondary lesions. These metastasis suppressors can interfere with any step of the metastatic cascade, including invasion, angiogenesis, migration and homing, intravasation, survival and proliferation in a new environment [3]. KISS1 was originally identified in experiments in which transfer of human chromosome 6 to metastatic melanoma cells suppressed metastasis in a mouse xenograft model $[1,2]$. The gene was identified by subtractive hybridization and differential display and was sufficient to suppress the metastatic phenotype of several human cell lines including breast cancer [4] and ovarian carcinoma [5] in mouse xenograft experiments. In clinical studies, the absence or reduced expression of KISS1 has been linked to higher grade and/or poor prognosis in several malignancies including melanoma $[6,7]$, carcinoma of the ovary $[8,9]$, stomach $[10,11]$, urinary bladder [12] esophagus [13], lung [14] and pancreas $[15,16]$.

KISS1 encodes a secreted protein that is sequentially proteolytically processed to generate multiple polypeptides ranging from 54 to 10 amino acids called kisspeptins (KP) that share the same amidated C-terminus [17-20]. Although other peptidic fragments are generated during this maturation process, the biological activity of KISS1 is thought to reside with KP. Their action is mediated through binding to and activation of GPR54, a G protein-coupled receptor (GPCR), also known as AXOR12 and hOT7T175 and coupled 
to $\mathrm{G \alpha}_{\mathrm{q}}$ [17-20]. GPR54 activation by kisspeptins was shown to block chemotaxis to fetal bovine serum (FBS), activate extracellular signal-regulated kinase (ERK1/2), induce formation of stress fibers, phosphorylate focal adhesion complex, decrease expression of matrix metalloproteinase 9 (MMP-9) and reduce cell proliferation in receptor transfectants $[17,19,21]$. We have shown previously that activation of GPR54 by kisspeptin-10 (KP10) inhibited chemotaxis induced by stromal cell derived factor 1 (SDF-1)/CXCL12 and its receptor CXCR4 [22], a mechanism shown in several models to be involved in the migration of CXCR4+ tumor cells to the organs that secrete its ligand such as the lungs, liver, brain and bones [23]. Activation of GPR54 suppressed Akt phosphorylation by CXCR4 [22]. We recently demonstrated a number of cellular events resulting from GPR54 activation, including suppression of the phosphorylation of Akt following activation of their respective receptor tyrosine kinase (RTK) by epidermal growth factor (EGF) and insulin, induction of apoptosis, expression of TNF- $\alpha$ [24], as well as profound modifications of the actin cytoskeleton through RhoA and Rhoassociated kinase (ROCK) activation [25].

The human melanoma cell line C8161.9 was used for the initial description of the anti-metastatic properties of KISS1 [1]. In xenograft experiments in nude mice, the secretion of KP by a C8161.9 clone was shown to have a remarkable capacity to reduce metastasis to the lungs, eyes, bones and kidneys by keeping tumor cells quiescent after dissemination and seeding in secondary organs [26]. Furthermore, this model is especially interesting in that, although the metastatic growth of C8161.9 cells is suppressed by the secretion of KPs, the cells do not express GPR54 [26]. This suggests that KPs have the capacity to act on the microenvironment of tumor cells rather than on 
the tumor cells themselves [27]. This property could establish KPs or synthetic analogs as prime candidates as a therapeutic option for the treatment of metastatic disease as it could be used even after dissemination from the primary tumor has occurred, a property that would be especially desirable for the treatment of melanoma. To test this hypothesis, we used a recently developed analog of KP with vastly improved stability in order to inhibit metastatic growth of C8161.9 cells. The results of these experiments prompted us to revisit the C8161.9 xenograft model. We found that the remarkable anti-metastatic properties of KISS1 in that model rely largely on an artifact of clonal selection rather than on KISS1 expression.

\section{Methods}

Cell lines and transfections: Parental C8161.9, C8161.9 transfected with an empty pcDNA3 plasmid, selected with G418 and then transduced with a lentivirus encoding EGFP (C8161.9-pc3) and C8161.9 tranfected with pcDNA3 coding for KISS-FLAGMetastin (KFM, a version of KISS1 incorporating a FLAG tag), cloned and then transduced with a lentivirus encoding EGFP (C8161.9-KFM) were previously described [26]. Whereas C8161.9-pc3 cells are a polyclonal population derived from the parental cells by antibiotic selection (G418), C8161.9-KFM cells are a single cell clone obtained by antibiotics selection (also G418) followed by limiting dilution and screening for secretion of KISS1. These different cell lines were kindly provided by Dr. Danny Welch (UAB, Birmingham, AL).

The open reading frame coding for KFM was amplified by PCR from the DNA purified from C8161.9-KFM cells and subcloned in pcDNA3.1 (Invitrogen) with G418 or 
hygromycin selection marker. Correct sequence was verified by Sanger sequencing. Parental C8161.9 cells (for pcDNA3.1-G418) and C8161.9-pc3 (for pcDNA3.1-Hygro) were transfected using Lipofectamine complemented with LTX reagent (Invitrogen, Carlsbad, CA) according to manufacturer's instructions and selected with the appropriate antibiotics for 2 weeks before cloning.

KP analogs: FTM145 ((4-fluorobenzoyl)-Phe-Gly=Leu-Arg-Trp-NH2) and FTM145carboxylate (non-amidated) were previously described [28]. Lyophilyzed compounds were dissolved in DMSO at $12.5 \mathrm{mM}$, diluted in PBS at $250 \mu \mathrm{M}$ and sterile filtered. Single-use aliquots were prepared for in vivo experiments.

Western blotting: Western blotting was performed as previously described [22]. Briefly, transfectants of 293 cells with stable expression of GPR54 were seeded in $35 \mathrm{~mm}$ plates, starved overnight in serum-free medium, then exposed to log dilutions of KP10, FTM145 or FTM145-carboxylate for 5 min. Cell lysates were prepared in SDS sample buffer. Western blots were incubated first with antibodies to total ERK1/2 or phospho-ERK1/2 (Cell Signaling Technology, Danvers, MA), then with secondary antibodies labeled with peroxidase (Jackson ImmunoResearch, West Grove, PA). Western blots were finally incubated with a chemiluminescent substrate (ECL-Plus, Amersham, GE-Healthcare, Piscataway, NJ) and analyzed with a LAS-4000 digital imaging system (GE-Healthcare).

Soft agar assay: A bottom layer consisting in $5 \mathrm{~mL}$ of $2 \mathrm{x}$ Dulbecco's Modification of Essential Medium/Ham's F-12 (DMEM/F-12) mixed at $40^{\circ} \mathrm{C}$ with $5 \mathrm{~mL}$ of a $1 \%$ solution 
of agar noble (BD Biosciences, Sparks, MD) in $\mathrm{H}_{2} \mathrm{O}$ and layered in a $100 \mathrm{~mm}$ tissue culture dish. For the top layer, $1.5 \times 10^{4}$ cells were added to $5 \mathrm{~mL}$ of $2 \times \mathrm{DMEM} / \mathrm{F}-12$ mixed with $5 \mathrm{~mL}$ of a $0.7 \%$ solution of agar in $\mathrm{H}_{2} \mathrm{O}(0.35 \%$ final concentration of soft agar). After solidification, $10 \mathrm{~mL}$ of complete liquid medium was added. Medium was replaced every 3 days for 4 weeks. Colonies were photographed using an inverted microscope or an ImageQuant LAS 4000 digital imaging system (GE Healthcare, Piscataway, NJ). For clone isolation, colonies were extracted using $1000 \mu \mathrm{L}$ sterile tips and placed in warm trypsin-EDTA in medium. Repeated pipeting was used to dissociate the colonies and the cells were plated in tissue culture dishes in complete growth medium.

Detection and quantification of secreted KFM by ELISA: Conditioned media were prepared by seeding $5 \times 10^{5}$ cells in $2 \mathrm{~mL}$ of complete growth medium in $35 \mathrm{~mm}$ dishes. Cells were grown for 48 hours at $37^{\circ} \mathrm{C}$ in $5 \% \mathrm{CO}_{2}$. Conditioned media were then collected and cleared by centrifugation at 2,000 g for 5 min. ELISA plates (Maxisorp, Nalge-Nunc, Rochester, NY) were coated for $1 \mathrm{~h}$ at room temperature (RT) with $100 \mu \mathrm{L}$ a mouse monoclonal antibody specific for KISS1 (clone 1A5 or 6A4, kindly provided by Dr. MaryAnn Accavitti-Loper, UAB, Birmingham, AL) diluted at $10 \mu \mathrm{g} / \mathrm{mL}$ in PBS. After blocking for $1 \mathrm{~h}$ with TBS containing 5\% Bovine Serum Albumin (BSA), antigen capture was performed with $200 \mu \mathrm{L}$ of conditioned medium for $1.5 \mathrm{~h}$ at RT, followed by detection with M2 anti-FLAG antibody conjugated to peroxidase (Sigma, St Louis, MO) diluted 1:5000 in TBS 1\% BSA. The substrate (200 $\mu$ L, SigmaFast, Sigma) was added for $30 \mathrm{~min}$ and the reaction was stopped with $50 \mu \mathrm{L}$ of $1 \mathrm{~N} \mathrm{HCl}$. Absorbance was 
measured at $490 \mathrm{~nm}$ using a spectrophotometer (BioTek, Winooski, VT). Background was determined using a conditioned medium obtained with C8161.9-pc3 cells and was identical to the signal obtained with TBS-BSA alone.

Detection of intracellular KISS1 by flow cytometry: Cells were detached with Trypsin-EDTA, fixed in PBS containing 4\% paraformaldehyde (PFA) for $1 \mathrm{~h}$ at RT, washed 4 times in PBS, then permeabilized for 5 min in PBS containing $0.1 \%$ Triton X100. PFA was quenched with $100 \mathrm{nM}$ glycine in PBS for $30 \mathrm{~min}$, followed by blocking in PBS containing $1 \%$ BSA and $10 \%$ goat serum for $1 \mathrm{~h}$ at RT. Cells were incubated with the 1A5 anti-KISS1 antibody or a control IgG (SouthernBiotech, Birmingham, AL) (both $2 \mu \mathrm{g} / \mathrm{mL}$ in PBS $1 \%$ BSA $10 \%$ goat serum) for $90 \mathrm{~min}$ at RT, followed by overnight incubation at $4^{\circ} \mathrm{C}$ with $\mathrm{F}\left(\mathrm{ab}^{\prime}\right) 2$ goat anti-mouse $\operatorname{IgG}$ labeled with phycoerythrin (10 $\mu \mathrm{g} / \mathrm{mL}$, Jackson ImmunoResearch, West Grove, PA). Samples were analyzed on a FACS Sort flow cytometer and Cell Quest software (BD Biosciences, San Jose, CA).

Quantitative real-time PCR (qPCR): Total RNA was extracted from cell lines using the mirVana miRNA isolation kit (Ambion, Austin, TX) and quality was assessed using a RNA 6000 Nano kit and a Bioanalyser 2100 (Agilent Technologies, Santa Clara, CA). For cDNA synthesis, 500 ng of RNA was used with the Enhanced Avian First Strand Synthesis kit (Sigma). QPCR was performed on a Lightcycler 480 (Roche, Indianapolis, IN) with HotStartIT SYBR Green qPCR mastermix (USB, Cleveland, OH) using the following primers: Forward 5'-actcactggtttcttggcagc-3', Reverse 5'-accttttctaatggctccca$3^{\prime}$. Cycling conditions were $95^{\circ} \mathrm{C}$ for $3 \mathrm{~min}, 40$ cycles of $95^{\circ} \mathrm{C}$ for $10 \mathrm{sec}, 60^{\circ} \mathrm{C}$ for $20 \mathrm{sec}$ 
$72{ }^{\circ} \mathrm{C}$ for $30 \mathrm{sec}$, followed by a melting curve from $45{ }^{\circ} \mathrm{C}$ to $95{ }^{\circ} \mathrm{C}$. Standard curve was obtained using serial dilutions of the pcDNA3.1-KFM plasmid. Jurkat cells transfected with GPR54 were used as a negative controls because the presence of very low concentrations of KP was shown to induce apoptosis in these cells.

In vivo experiments: 3-4 weeks old Nude mice (Harlan, Indianapolis, IN) were used for all experiments. For orthotopic tumor formation, mice were injected intradermally with 2 x $10^{5}$ cells in $50 \mu \mathrm{L}$ Hank's balanced salt solution (HBSS) using a Hamilton syringe and a 30-gauge needle. Mice were euthanized after 5 weeks and tumor size was measured using a caliper. For metastatic tumor formation, 5 x $10^{5}$ cells were injected in the lateral tail vein in $100 \mu \mathrm{L}$ of HBSS using an insulin syringe and a 31-gauge needle. Antimetastatic activity of FTM145 was assessed by injecting $150 \mu \mathrm{L}$ of a $250 \mu \mathrm{M}$ solution diluted in PBS, an amount expected to generate an estimated peak concentration of about $20 \mu \mathrm{M}$ in the extracellular space in 15-20 gram animals. Mice were euthanized after 5 weeks or when moribund for survival experiments. All experiments were conducted in accordance with the guidelines of the Institutional Animal Care and Use Committee at Thomas Jefferson University. Animals were dissected and lungs were fixed in PBS containing 4\% PFA for 24 hours before being processed for histology studies. Sections were stained with hematoxylin and eosin.and scanned using a ScanScope XT scanner (Aperio Technologies, Vista, CA). Images were visualized with Spectrum software (Aperio). 
Comparative Genomic Hybridization (CGH) array: $0.5 \mu \mathrm{g}$ of DNA from cell lines, and Female control gDNA (Promega, Madison, WI) were digested by AluI and RsaI restriction enzymes, then labeled with $\mathrm{Cy} 5$ and $\mathrm{Cy} 3$ respectively using the Enzymatic Labeling kit from Agilent according to the manufacturer's protocol. Samples were combined, heat-denatured and mixed with COT-1 DNA (Roche), blocking buffer and HiRPM buffer (both from Agilent) before being hybridized to an Agilent $4 \mathrm{x} 44 \mathrm{k}$ human $\mathrm{CGH}$ microarray for $24 \mathrm{~h}$ at $65^{\circ} \mathrm{C}$. Washing was performed as recommended by the vendor, and the arrays were scanned using an Agilent DNA Microarray Scanner. Data were processed with Feature extraction Software version 10.7.3.1 and analyzed in DNA Analytics version 4.0.76 (Agilent Technologies)

Statistics: Averages of measureable variables were compared between experimental groups using Student's $t$-test with a 95\% confidence interval. $\mathrm{P}$ values $<0.05$ were considered significant. The survival experiment was analyzed using the Kaplan-Meier method. All analyses were performed with Prism 3.0 (GraphPad. La Jolla, CA).

\section{Results}

\section{Injection of a KP analog does not reduce metastasis of C8161.9 cells.}

We used an analog of KP to inhibit the metastatic growth of C8161.9 cells in nude mice to determine whether pharmacologic administration of KP could reproduce the antimetastatic effect seen when expression was programmed in tumor cells. Using the same xenograft model, the secretion of KP by clones of C8161.9-KFM cells was previously shown to inhibit metastasis to the lungs by more than $90 \%$ [26]. This established model 
used the polyclonal C8161.9-pc3 cells as positive control for metastasis. Both pc3 and KFM cells expressed EGFP to allow ex vivo localization of tumor cells in organs. We selected the analog FTM145 because of its resistance to proteolytic cleavage and its extended half-life in mouse serum [28]. We first confirmed that the injectable solution of the C-amidated version of FTM145 was fully active in vitro. Serial dilutions of KP10, FTM145 and the barboxylated version of FTM145 were added to HEK-293 cells expressing GPR54 and cell lysates were analyzed by western blotting for activation of ERK1/2. KP10 and FTM145 were equally potent, with full phosphorylation of ERK achieved at $1 \mathrm{nM}$ of each ligand. In contrast, the non-amidated FTM-145 only induced significant activation of GPR54 at $1 \mu \mathrm{M}$ (Figure 1A).

Two groups of 20 nude mice each were injected on day 1 with metastatic C8161.9pc3 cells (i.v.). FTM145 (amidated, $n=20)$ or PBS alone $(\mathrm{n}=20)$ were administered daily through subcutaneous injection. In this survival experiment, FTM145 did not provide any advantage compared to injection of PBS (Figure 1B). Upon dissection, all animals showed extensive metastasis to the lungs (comparable to the animal shown in Figures $3 \mathrm{~B}$ and $3 \mathrm{C}, \mathrm{pc} 3$ panels), irrespectively of the treatment received, indicating that injection of a KP mimetic was incapable of inhibiting metastasis of C8161.9.

\section{C8161.9-KFM cells differ phenotypically from original C8161.9 cells}

In an attempt to reconcile the data showing the absence of in vivo activity of FTM145 with the previously published data showing the strikingly reduced metastatic potential of C8161.9 cells secreting KPs, we conducted a series of experiments comparing the in vitro and in vivo phenotypes of different types of C8161.9 cells: 1) 
parental C8161.9 cells, 2) C8161.9 transfected with an empty pcDNA3 plasmid, selected with G418 and then transduced with a lentivirus encoding EGFP (C8161.9-pc3) and 3) C9161.9 tranfected with pcDNA3 coding for KFM, cloned and then transduced with a lentivirus encoding EGFP (C8161.9-KFM). C8161.9-pc3 and C8161.9-KFM were used in previously published in vivo experiments [26] and C8161.9-pc3 were used as metastatic cells throughout the current study in order to remain consistent with published work.

Whereas both parental C8161.9 (not shown) and C8161.9-pc3 (Figure 2A) consisted of a vast majority of small cells and a minority of larger cells, C8161.9-KFM were much larger and very homogeneous in size and morphology (Figure 2A). The 3 cell types were tested for their capacity to form colonies in soft agar. In 3 separate experiments, C8161.9-pc3 cells did not form any colonies, in contrast with C8161.9KFM cells which consistently established large numbers of colonies (Figure 2B). The parental C8161.9 cells formed a few colonies, but these colonies were similar in size to those made by KFM cells. Seven colonies (most likely clones, each one originating from a single cell based on the distance separating the colonies as well as their spherical geometry) were isolated from the soft agar plate with a pipet tip and expanded in culture. All 7 clones (agar clones AC1-7) were composed of large cells morphologically similar to C8161.9-KFM cells, as shown in Figure 2A for AC4 and AC5. After expansion, these clones maintained their capacity to form colonies in soft agar, AC5 being as efficient as KFM cells (Figure 2C). All cell types displayed the same growth rate in normal culture condition with a doubling time of 24 hours (not shown). These data suggest that the parental C8161.9 cells contain multiple populations with varying behaviors, including a 
few larger cells capable of establishing colonies in soft agar. It appears these cells were preferentially selected in the cloning process leading to the isolation of C8161.9-KFM cells and also seem to have been depleted (partially or completely) during the selection of the C8161.9-pc3 cells.

C8161.9-KFM cells were previously described as being able to establish orthotopic tumors with an efficiency similar to C8161.8-pc3 cells [26]. However, 4 weeks after intradermal inoculation of $2 \times 10^{5}$ cells, the average size of the tumors formed by C8161.9-KFM $\left(19 \pm 17 \mathrm{~mm}^{3}, \mathrm{n}=3\right)$ cells was less than $10 \%$ of the size of tumors made by $\mathrm{C} 8161.9-\mathrm{pc} 3$ cells $\left(400 \pm 40 \mathrm{~mm}^{3}, \mathrm{n}=3\right)$ and even several times smaller than tumors made after inoculation of $2 \times 10^{4} \mathrm{pc} 3$ cells $\left(137 \pm 81 \mathrm{~mm}^{3}, \mathrm{n}=3\right)$ (Figure 3A). To test the possibility that local secretion of $\mathrm{KP}$ is responsible for that reduction in growth potential, we performed an experiment in which $2 \times 10^{5} \mathrm{KFM}$ cells were mixed with $2 \times 10^{4} \mathrm{pc} 3$ cells and injected intradermally. The average volume of the tumors made by this mixed population $\left(198 \pm 92 \mathrm{~mm}^{3}, \mathrm{n}=3\right)$ was not significantly different from the volume of tumors made by $2 \times 10^{4} \mathrm{C} 8161.9 \mathrm{pc} 3$ cells alone (Figures 3A), indicating that, despite an initial numerical excess, C8161.9-KFM cells were unable to inhibit the growth of C8161.9-pc3 cells.

\section{Soft agar clones reproduce the phenotype of C8161.9-KFM cells}

After having established that the soft agar clones obtained from the original C8161.9 cells behaved similarly to KFM cells in vitro, we conducted experiments to test whether they also exhibited a decreased metastatic potential. Nude mice $(n=4$ for each cell type) received an i.v. injection of C8161.9-pc3, C8161.9-KFM or one of 5 soft agar 
clones (AC2, 3, 4, 5 and 7). After 5 weeks, mice injected with pc3 cells were visibly sick whereas all other mice appeared completely healthy. Upon dissection, mice injected with pc3 cells showed extensive metastasis to the lungs (Figure 3B and Table 1). All other cell types showed a dramatically reduced metastasis with no or very few visible lesions (Figure 3B). Lungs of all animals were fixed in PFA and sections were stained with hematoxylin and eosin. Analysis of these sections confirmed the dramatic reduction of metastasis observed with the KFM cells and AC2, 3, 4, 5 and 7. Representative fields of sections are shown in Figure 3C. To rule out the possibility that the decrease of metastatic potential by these clones was due to endogenous expression of KISS1, we performed qPCR. KISS1 mRNA could be detected at high levels in the transfected KFM cells but was absent or expressed at very low level in the soft agar clones (Figure 3D) as well as Jurkat cells transfected with GPR54 that do not express KISS1 and were shown to be extremely sensitive to exogenous KP10 [24]. These data indicate that clonal selection in soft agar did not select for cells overexpressing KISS1. These data indicate that a minor subpopulation of cells present in the parental C8161.9 cell line phenotypically resembles C8161.9-KFM cells (both in morphology and in tumor-forming potential) without expressing KISS1. They also illustrate an inverse relationship between the clonogenicity in soft agar and the capacity to form tumors in nude mice.

\section{Single cell cloning of C8161.9 cells is biased toward large cells similar to KFM cells.}

Since the KP-expressing cells already available displayed a phenotype that did not represent the original $\mathrm{C} 8161.9$ cell line, we sought to prepare new transfectants expressing KISS1 using parental C8161.9 cells or C8161.9-pc3 cells. In order to remain 
as close as possible to the original model, we designed two parallel strategies. First, in order to derive new transfectants from cells with a tested and well established metastatic potential, we used as host the C8161.9-pc3 transfectants that have previously been used in animal experiments, including those described above. Since these cells were already resistant to G418, we used the hygromycin pcDNA3 vector to stably introduce KFM in these cells. In the second strategy, we established new transfectants from the parental C8161.9 cell line using G418 as a selection marker in order to exactly reproduce the conditions that presided over the creation of the pc3 and KFM cells described and employed previously. Since every single cell injected to the animals for the xenograft studies must secrete KISS1, antibiotics selection is not sufficient and single cells cloning is required to guarantee homogeneity of the cell population. Cells were transfected with pcDNA3.1-KFM-G418 (for parental C8161.9) or pcDNA3.1-KFM-Hygro (for C8161.9pc3), both plasmids containing an insert coding for KFM identical to that employed in Nash et al. [26]. After selection with G418 (for parental cells) or hygromycin (for C8161.9-pc3 cells), stable KFM transfectants were obtained by single cell cloning. We first used limiting dilution in 96-well plates, which produced only 2 clones out of 6 plates (576 wells) for the parental cells and 3 clones out of 12 plates (1152 wells) for the pc3 cells. Each of these clones consisted of large cells similar to the C8161.9-KFM cells used previously (not shown). The very low number of clones obtained and the recurring "large cell" phenotype indicate that C8161.9 cells are very difficult to clone and that standard protocols are heavily biased toward the selection of large cells that seem to be the only ones capable of growing at very low density. Because this bias increased the risk of 
selecting cells that functionally did not represent the C8161.9 cell line as a whole, these few clones were discarded without further study.

To avoid this problem and obtain multiple clones representing the diversity of C8161.9 cells, we used a "feeder cell" approach in which transfected cells (10 cells / mL) were mixed with non-transfected C8161.9 cells $\left(1 \times 10^{4} / \mathrm{mL}\right)$ before seeding $(100 \mu \mathrm{L}$ /well), so that transfectants could establish supportive contacts with neighboring cells. After 6 days of co-culture in complete medium without selection antibiotics, cells were about $50 \%$ confluent. At this point, G418 or hygromycin was re-introduced in the growth medium, eliminating all the non-transfected cells in 2-3 days. Resistant clones were left to expand and were detected under an inverted microscope. Supernatants were collected and tested by ELISA for presence of secreted KFM. More than 125 clones were obtained from five 96-well plates, indicating that this protocol allowed the isolation of positive clones with no or minimum selective pressure other than the selection antibiotic. The clones displayed a variety of morphologies, most consisting of small cells and a few composed of large cells, thus demonstrating the heterogeneity of the original cell line (not shown).

Clones positive for secretion of KFM (over 40) were expanded and further tested for expression of KFM by flow cytometry and semi-quantitative ELISA. Flow cytometry allowed the selection of genuine single cell clones with homogeneous expression of intracellular KFM (Figure 4A). Some clones displayed expression as high as the original C8161.9-KFM clone. Secretion of KFM was assessed by semi-quantitative ELISA. Representative clones are shown in Figure 4B. 


\section{New C8161.9-KFM clones show normal metastatic potential}

The metastatic potential of the new C8161.9 clones expressing high levels of KFM was evaluated in nude mice. A total of 17 clones were tested, either as individual clones ( 8 clones) or as pools of 2 to 4 clones ( 9 clones in 3 pools) (Table 1). Two or 4 nude mice were injected intravenously with $5 \times 10^{5}$ cells and were sacrificed after 5 weeks. The lungs of mice injected with large cell variants (clones $2 \mathrm{E} 8$ and 5C6) showed strongly decreased metastasis compared to mice injected with C8161.9-pc3 cells, whereas mice injected with clones composed of small cells that resembled the parental line showed extensive metastasis similar to that obtained with pc3 cells, independently of the KFM expression level (Figure 4C and Table 1). Although the number of animals used for each clone is small, data were very consistent for each clone and within each phenotype (small vs. large cells). Only one small cell clone (4F12) showed a decreased number of metastatic lesions (although most lesions were large) (Figure 4C). This data demonstrate that the C8161.9 cell line consists of multiple subpopulations with different metastatic potential and that individual clones can exhibit in vivo clonogenic activities resulting in a metastatic potential ranging from very high to very low, independently of KP expression.

\section{Morphological differences correlate with genomic changes in C8161.9 cells evident following selection.}

Having established a correlation between cell morphology and cell phenotype in vitro and in vivo, we hypothesized that this phenotypic heterogeneity could also be evidenced at a genetic level. We used CGH with high-resolution DNA microarrays to detect DNA copy number variations (CNV; gains and losses) in nine different C8161.9 
variants: parental C8161.9, C8161.9-pc3 and the new KFM clone 6F10 for the small cell / highly metastatic category, C8161.9-KFM (the original clone used in Nash and colleagues), and soft agar clones AC2, 3, 4, 5 and 7 for the large cell / poorly metastatic category. Data analysis indicated that, although some CNV shared by all cell types from both groups (gain of chromosome 3q, loss of $9 p$ and $17 q$ ) indicate a common origin, members of the large cell group shared additional CNV that were absent in small cells and could not be detected in parental C8161.9 cells or C8161.9-pc3 cells, such as loss of chromosomes 4, 5p, 5q, 9p and 17p, as well as gain of chromosomes 9q and 17q (Figure 5). The fact that these CNV could not be detected in the parental cell line correlates with the fact that the large majority of the cells in the C8161.9 cell line consists of small cells and that the large cell variants represent a minor population. These data also show that the C8161.9-KFM clone used in the previous study differed from the original C8161.9 cell line at several genomic and phenotypic levels in a manner unrelated to KISS1 expression.

\section{Discussion}

Among the members of the family of metastasis suppressors, expression of KISS1 was shown to confer dramatic inhibition of the post-migratory step of the metastatic process. The initial studies for characterizing the mechanisms responsible for the antimetastatic effect assumed an autocrine mechanism of action whereby KP secreted by tumor cells would bind and activate GPR54 on the same cells, thereby inhibiting cell migration as shown with the B16 model of melanoma [19]. The C8161.9 model indicated instead that expression of GPR54 on the tumor cells was not required for KISS1 
suppression of metastasis formation [26]. The observation that secretion of KISS1 by C8161.9 melanoma cells maintained the cells in a prolonged state of dormancy opened new perspectives toward the therapeutic use of KP molecular analogs that could be used to treat metastatic disease even after the initial spread of tumor cells. Since these findings also suggested that the target of KISS1 was the microenvironment, the C8161.9 model represented an appropriate system to investigate this hypothesis. However, administration of high concentrations of a fully active KP10 analog with vastly improved resistance to proteolytic degradation failed to reduce metastasis of C8161.9 cells in nude mice. Also, C8161.9-KFM established orthotopic tumors much more slowly than control C8161.9pc3 cells and failed to inhibit the growth of pc3 when locally co-injected with a 10:1 excess. Although these data could be explained (insufficient local concentration of the analog, action of KISS1 mediated by a proteolytic fragment other than KP, KFM cells quickly outgrown by pc3 cells in the mixing experiment), they together prompted us to reconsider the validity of the C8161.9 model.

Here we present evidence that the C8161.9-KFM previously described as being poorly metastatic shared in vitro features with a cell population present at low frequency (7 clones obtained in soft agar culture from $1.5 \times 10^{4}$ cells) that could be derived from the parental cell line. Although it also exhibited some level of internal heterogeneity, as a whole, this sub-population consisted of larger cells that had the capacity to form colonies in soft agar, could grow as single cells in vitro, had the same in vitro growth rate as smaller cells, but grew very poorly in vivo when grafted in nude mice, even in the absence of KISS1 expression. By contrast, the predominant population of C8161.9 cells were smaller, did not form colonies in soft agar, could not be cloned using standard 
limiting dilution protocols, but were highly tumorigenic in mice. Based on these differences in growth properties, standard selection procedures using selectable markers and single cell cloning favor the outgrowth of the non-metastatic population. These two distinct cell populations (large and mostly small cells) also segregate in two different categories at the genomic level, thus mirroring and substantiating the phenotypic differences. The very strong bias toward the selection of large non-metastatic / nontumorigenic cells during the cloning process is most likely the reason for the previous description of the anti-metastatic effect of the secretion of KFM. This was confirmed by showing that multiple new clones obtained without the bias of that selective pressure and expressing high levels of KISS1 maintained the metastatic potential of the parental cell line.

Our data do not necessarily disprove that KISS1 is a metastasis suppressor in melanoma and other cancers, even though its role in the C8161.9 xenograft model appears to be unclear. Indeed, numerous studies investigating the expression of KISS1 and GPR54 in human samples suggest that this system plays a role in a number of malignancies, although a more limited one that what was suggested by the C8161.9 model. Some studies also support the hypothesis of an effect of KISS1 on the microenvironment, especially on angiogenesis. In particular, KISS1 was shown to trigger vasoconstriction by acting on smooth muscle cells [29]. Another recent study indicated KISS1 signaling in endothelial cells and the resulting inhibition of angiogenesis [30]. The results presented here show that the C8161.9 xenograft model does not constitute an appropriate system to investigate the mechanism of action of KISS1. They especially highlight the risk of experimental artifacts resulting from the selection of single cell 
clones from a cell line after transfection. When cloning is necessary - as it is in the case of selection based on the expression of a secreted factor - great care should be taken to characterize the clones to prove that they faithfully represent the original cell population, both in vitro and in vivo. In that respect, the high resolution $\mathrm{CGH}$ employed here represents a method of choice. 


\section{References:}

1. Welch DR, Chen P, Miele ME, McGary CT, Bower JM, Stanbridge EJ, et al. Microcell-mediated transfer of chromosome 6 into metastatic human C8161 melanoma cells suppresses metastasis but does not inhibit tumorigenicity. Oncogene 1994; 9: 255262.

2. Lee JH, Miele ME, Hicks DJ, Phillips KK, Trent JM, Weissman BE, et al. KiSS1, a novel human malignant melanoma metastasis-suppressor gene. J Natl Cancer Inst 1996; 88: 1731-1737.

3. Stafford LJ, Vaidya KS, Welch DR. Metastasis suppressors genes in cancer. Int J Biochem Cell Biol 2008; 40: 874-891.

4. Lee JH, Welch DR. Suppression of metastasis in human breast carcinoma MDAMB-435 cells after transfection with the metastasis suppressor gene, KiSS-1. Cancer Res 1997; 57: 2384-2387.

5. Jiang Y, Berk M, Singh LS, Tan H, Yin L, Powell CT, et al. KiSS1 suppresses metastasis in human ovarian cancer via inhibition of protein kinase $\mathrm{C}$ alpha. Clin Exp Metastasis 2005; 22: 369-376.

6. Martins CM, Fernandes BF, Antecka E, Di Cesare S, Mansure JJ, Marshall JC, et al. Expression of the metastasis suppressor gene KISS1 in uveal melanoma. Eye 2008; 22: 707-711.

7. Shirasaki F, Takata M, Hatta N, Takehara K. Loss of expression of the metastasis suppressor gene KiSS1 during melanoma progression and its association with LOH of chromosome 6q16.3-q23. Cancer Res 2001; 61: 7422-7425. 
8. Prentice LM, Klausen C, Kalloger S, Kobel M, McKinney S, Santos JL, et al. Kisspeptin and GPR54 immunoreactivity in a cohort of 518 patients defines favourable prognosis and clear cell subtype in ovarian carcinoma. BMC Med 2007; 5: 33.

9. Hata K, Dhar DK, Watanabe Y, Nakai H, Hoshiai H. Expression of metastin and a G-protein-coupled receptor (AXOR12) in epithelial ovarian cancer. Eur J Cancer 2007; 43: 1452-1459.

10. Dhar DK, Naora H, Kubota H, Maruyama R, Yoshimura H, Tonomoto Y, et al. Downregulation of KiSS-1 expression is responsible for tumor invasion and worse prognosis in gastric carcinoma. Int J Cancer 2004; 111: 868-872.

11. Guan-Zhen Y, Ying C, Can-Rong N, Guo-Dong W, Jian-Xin Q, Jie-Jun W. Reduced protein expression of metastasis-related genes (nm23, KISS1, KAI1 and p53) in lymph node and liver metastases of gastric cancer. Int J Exp Pathol 2007; 88: 175-183.

12. Sanchez-Carbayo M, Capodieci P, Cordon-Cardo C. Tumor suppressor role of KiSS-1 in bladder cancer: loss of KiSS-1 expression is associated with bladder cancer progression and clinical outcome. Am J Pathol 2003; 162: 609-617.

13. Ikeguchi M, Yamaguchi K, Kaibara N. Clinical significance of the loss of KiSS-1 and orphan G-protein-coupled receptor (hOT7T175) gene expression in esophageal squamous cell carcinoma. Clin Cancer Res 2004; 10: 1379-1383.

14. Zheng S, Chang Y, Hodges KB, Sun Y, Ma X, Xue Y, et al. Expression of KISS1 and MMP-9 in non-small cell lung cancer and their relations to metastasis and survival. Anticancer Res 2010; 30: 713-718. 
15. Masui T, Doi R, Mori T, Toyoda E, Koizumi M, Kami K, et al. Metastin and its variant forms suppress migration of pancreatic cancer cells. Biochem Biophys Res Commun 2004; 315: 85-92.

16. Nagai K, Doi R, Katagiri F, Ito T, Kida A, Koizumi M, et al. Prognostic value of metastin expression in human pancreatic cancer. J Exp Clin Cancer Res 2009; 28: 9.

17. Kotani M, Detheux M, Vandenbogaerde A, Communi D, Vanderwinden JM, Le Poul E, et al. The metastasis suppressor gene KiSS-1 encodes kisspeptins, the natural ligands of the orphan G protein-coupled receptor GPR54. J Biol Chem 2001; 276: 3463134636.

18. Bilban M, Ghaffari-Tabrizi N, Hintermann E, Bauer S, Molzer S, Zoratti C, et al. Kisspeptin-10, a KiSS-1/metastin-derived decapeptide, is a physiological invasion inhibitor of primary human trophoblasts. J Cell Sci 2004; 117: 1319-1328.

19. Ohtaki T, Shintani Y, Honda S, Matsumoto H, Hori A, Kanehashi K, et al. Metastasis suppressor gene KiSS-1 encodes peptide ligand of a G-protein-coupled receptor. Nature 2001; 411: 613-617.

20. Muir AI, Chamberlain L, Elshourbagy NA, Michalovich D, Moore DJ, Calamari A, et al. AXOR12, a novel human G protein-coupled receptor, activated by the peptide KiSS-1. J Biol Chem 2001; 276: 28969-28975.

21. Yan C, Wang H, Boyd DD. KiSS-1 represses 92-kDa type IV collagenase expression by down-regulating NF-kappa B binding to the promoter as a consequence of Ikappa Balpha -induced block of p65/p50 nuclear translocation. J Biol Chem 2001; 276: 1164-1172. 
22. Navenot JM, Wang Z, Chopin M, Fujii N, Peiper SC. Kisspeptin-10-induced signaling of GPR54 negatively regulates chemotactic responses mediated by CXCR4: a potential mechanism for the metastasis suppressor activity of kisspeptins. Cancer Res 2005; 65: 10450-10456.

23. Muller A, Homey B, Soto H, Ge N, Catron D, Buchanan ME, et al. Involvement of chemokine receptors in breast cancer metastasis. Nature 2001; 410: 50-56.

24. Navenot JM, Fujii N, Peiper SC. KiSS1 metastasis suppressor gene product induces suppression of tyrosine kinase receptor signaling to Akt, tumor necrosis factor family ligand expression, and apoptosis. Mol Pharmacol 2009; 75: 1074-1083.

25. Navenot JM, Fujii N, Peiper SC. Activation of Rho and Rho-associated kinase by GPR54 and KiSS1 metastasis suppressor gene product induces changes of cell morphology and contributes to apoptosis. Mol Pharmacol 2009; 75: 1300-1306.

26. Nash KT, Phadke PA, Navenot JM, Hurst DR, Accavitti-Loper MA, Sztul E, et al. Requirement of KISS1 secretion for multiple organ metastasis suppression and maintenance of tumor dormancy. J Natl Cancer Inst 2007; 99: 309-321.

27. Beck BH, Welch DR. The KISS1 metastasis suppressor: a good night kiss for disseminated cancer cells. Eur J Cancer 2010; 46: 1283-1289.

28. Tomita K, Oishi S, Ohno H, Peiper SC, Fujii N. Development of Novel GProtein-Coupled Receptor 54 Agonists with Resistance to Degradation by Matrix Metalloproteinase. J Med Chem 2008.

29. Mead EJ, Maguire JJ, Kuc RE, Davenport AP. Kisspeptins are novel potent vasoconstrictors in humans, with a discrete localization of their receptor, G proteincoupled receptor 54, to atherosclerosis-prone vessels. Endocrinology 2007; 148: 140-147. 
30. Cho SG, Yi Z, Pang X, Yi T, Wang Y, Luo J, et al. Kisspeptin-10, a KISS1derived decapeptide, inhibits tumor angiogenesis by suppressing Sp1-mediated VEGF expression and FAK/Rho GTPase activation. Cancer Res 2009; 69: 7062-7070. 
Table 1: Metastatic potential of C8161.9 clones correlates with morphology and genomic changes rather than with expression of KISS1

\begin{tabular}{|l|l|l|l|l|}
\cline { 2 - 5 } \multicolumn{1}{l|}{} & KISS1 & Morphology & Metastases & CGH type \\
\hline C8161.9 parental & - & small & & 1 \\
\hline C8161.9-pc3 & - & small & +++ & \\
\hline C8161.9-KFM & + & large & +- & 2 \\
\hline AC2 & - & large & + +- & 2 \\
\hline AC3 & - & large & - & 2 \\
\hline AC4 & - & large & +- & 2 \\
\hline AC5 & - & large & + & 2 \\
\hline AC7 & - & large & + & 2 \\
\hline 2E8 & + & large & +- & \\
\hline 5C6 & + & large & +- & \\
\hline 1A4 & + & small & +++ & \\
\hline 4F12 & + & small & ++ & \\
\hline 3H9 & + & small & +++ & \\
\hline 6B6 & + & small & +++ & \\
\hline 3B8 & + & small & ++++ & \\
\hline 6F10 & + & small & ++++ & 1 \\
\hline Pool 1 (2E7+5A2+1A5) & + & small & +++ & \\
\hline Pool 2 (3F4+1F2+5H2+4H11) & + & small & ++++ & \\
\hline Pool 3 (3A3+4G9) & + & intermediate & ++++ & \\
\hline
\end{tabular}

The amount of metastasis was measured using the Aperio digital pathology system and is indicated as follows: - : no visible metastasis; +/- : 0 or 1 very small metastatic lesion (less than 10 cells) per section; + : 1-5 small lesions (less than 20 cells) per section, 
representing less than $1 \%$ of the surface area of the lung tissue in the section; $++: 1-10$ lesions per section, representing in total less than $10 \%$ of the surface area; +++ : multiple lesions representing $10-25 \%$ of the surface area; ++++ : multiple lesions representing in total more than $25 \%$ of the lungs surface area (often more than 50\%). CGH types 1 and 2 refer to the typical profiles illustrated in Figure 5. 


\section{Figure legends}

Figure 1: Injection of a KISS1 mimetic does not inhibit metastasis of C8161.9 cells in xenograft experiments. A. KP10 and C-ter-amidated FTM145 activated GPR54 in HEK-293 transfectants with similar potency as demonstrated by phosphorylation of ERK1/2. B. Daily subcutaneous injections of FTM145 did not suppress metastasis in nude mice inoculated with C8161.9 cells and did not offer any survival benefit compared to PBS as shown by Kaplan-Meier analysis.

Figure 2: C8161.9-KFM cell clone differs morphologically and phenotypically from the majority of the parental cell line. A. C8161.9-pc3 cells and the parental cell line (not shown) consisted mostly of small cells, whereas the poorly metastatic C8161.9-KFM clone previously described was homogenously constituted of larger cells. Clones isolated in soft agar culture from the parental C8161.9 cell line shared similarly large size. B. C8161.9-KFM cells, but not C8161.9-pc3, developed large numbers of colonies in soft agar. In contrast, parental C8161.9 cells only formed 7 colonies that were isolated and amplified (AC1-7). Images were taken using an inverted microscope with 10x objective. C. After in vitro amplification, clones isolated in soft agar from parental C8161.9 cells maintained the capacity to for colonies in soft agar, with an efficiency ranging from moderate (AC4) to as high as C8161.9-KFM cells (AC5). Pictures of the whole plates were taken with a GE LAS4000 imaging system.

Figure 3: C8161.9 soft agar clones replicated the in vivo phenotype of C8161.9-KFM cells without KISS1 expression. A. C8161.9-KFM cells exhibited a strongly reduced 
capacity to form orthotopic (intradermal) tumors in nude mice and did not suppress the growth of co-injected C8161.9-pc3 cells. Tumors were measured 4 weeks post-injection. Indicated P values were calculated using Student's $t$-test with a 95\% confidence interval. B and C. AC clones showed a reduced capacity to form lung metastases similar to C8161.9-KFM cells. Mice were injected in the tail vein with C8161.9-pc3, C8161.9$\mathrm{KFM}, \mathrm{AC} 2, \mathrm{AC} 3, \mathrm{AC} 4, \mathrm{AC} 5$ or AC7 cells. After 5 weeks, lungs showed extensive metastasis in mice inoculated with pc3 cells but no or very few (arrows) visible metastatic lesions in mice inoculated with KFM or AC cells (B) Images are from one representative mouse out of 4 for each clone. Sections of the lungs confirmed that both the number and the size of metastatic lesions (small lesions indicated by arrows) were strongly reduced with KFM or AC cells compared to pc3 cells. Boxed regions of the lowmagnification pictures are shown in the right panel. Representative fields out of 4 slides for 4 identical mice for each clone. D. Inhibition of the metastasis potential of AC cells is not related to endogenous KISS1 expression as demonstrated by the absence of detection of KISS1 transcripts by qPCR.

\section{Figure 4: Metastatic potential of new C8161.9 clones is independent from KISS1}

expression. New clones of C8161.9 cells expressing KFM displayed a wide range of metastatic potential related to cell morphology but independent of KFM expression. Multiple new C8161.9 single cell clones were derived after transfection with pcDNA3.1KFM and limiting dilution using non-transfected feeder cells. A. These new clones were tested by flow cytometry for expression of intracellular KFM using a mAb specific for KISS1 (1D5). The histograms show for each clone a single population with a staining 
intensity similar to the previously described C8161.9-KFM cells. B. Semi-quantitative ELISA for KFM secretion was performed on conditioned media obtained with new KFM clones (shown: clones 2E8, 4F12, 5G9, 1A4, 6F10 and 6B6) in comparison to 2 conditioned media obtained with the original C8161.9-KFM clone previously described. Results showed similar levels of secretion of KFM for the different clones. C. Representative sections of lungs from mice inoculated in the tail vein with new KFM clones and collected after 5 weeks. Clones consisting of large cells similar to KFM cells (e.g. 2E8) had strongly reduced metastasis whereas clones consisting of smaller cells similar to pc3 cells (e.g. 6F10) maintained a metastatic potential similar to C8161.9-pc3 cells. Clone 4F12 had intermediate metastatic potential with fewer but large metastatic lesions. Boxed regions of the low-magnification pictures are shown in the right panel.

Figure 5: Cell morphology and phenotype correlate with genomic changes among C8161.9 cells. CGH microarray analysis was performed on small cell clones (e.g. 6F10), large cell clones (e.g. original C8161.9-KFM and AC2), C8161.9-pc3 cells and the parental C8161.9 cell line. Data analysis revealed the strong similarity in CNV between large cells as well as the similarity between small cells, pc3 cells and the parental cell line. However, large cells diverged from small cells, pc3 and parental C8161.9. Chromosomes 3, 4, 5, 9 and 17 were the most discriminating. Losses (deletions) are indicated in green and gains (amplifications) are indicated in red. 